\title{
A sistematização da assistência de enfermagem desenvolvida para um caso raro de Kérion Celsi: relato de experiência
}

\author{
The systematization of nursing care developed for a rare case of Kerion Celsi: experience \\ report
}

La sistematización de la atención de enfermería desarrollada para un caso raro de Kerion de Celso: informe de experiencia

Brenda Ramos de Souza ${ }^{1 *}$, Flávia Daniele de Alencar Medeiro ${ }^{1}$, Suellen Cristhine Vital Guimarães², Tallyta Oliveira da Silva², Alan Rogério de Oliveira Ferreira², Natacha Mariana Farias da Cunha².

\section{RESUMO}

Objetivo: Relatar a construção do processo de enfermagem, por meio da Sistematização da Assistência de Enfermagem desenvolvida pela equipe de enfermeiros da pediatria para um caso raro de Kérion Celsi, desde sua admissão até a transferência do mesmo para resolução do caso. Relato de experiência: A primeira etapa para Sistematização de assistência de enfermagem consistiu na coleta de dados, seguida do exame físico realizado através de um formulário hospitalar. A partir dele, definiu-se os problemas ativos e potenciais que conduziram na definição de 6 diagnósticos de enfermagem adaptados à pediatria e intervenções voltadas a atuação nos problemas levantados, sendo a avaliação discutida e revisada diariamente através de uma evolução padronizada na Clínica pediátrica. Considerações finais: Neste relato, as etapas seguidas para realização do processo de enfermagem, através da sistematização da assistência de enfermagem, foram importantes para uma intervenção adequada, apesar de adaptada, para o caso em questão, necessitando de novos estudos para adequação da sistematização direcionada à assistência em enfermagem pediátrica.

Palavras-chave: Processo de enfermagem, Enfermagem pediátrica, Tinha do couro cabeludo.

\section{ABSTRACT}

Objective: To report the construction of the nursing process, through the Systematization of Nursing Care developed by the team of pediatric nurses for a rare case of Kerion Celsi, since its admission until the transfer of it for resolution of the case. Experience report: The first step towards systematization of nursing care consisted of data collection, followed by a physical examination performed using a hospital form. Based on it, the active and potential problems that led to the definition of 6 nursing diagnoses adapted to pediatrics and interventions aimed at acting on the problems raised were defined, the evaluation being discussed and reviewed daily through a standardized evolution in the Pediatric Clinic. Final considerations: In this report, the steps followed to carry out the nursing process, through the systematization of nursing care, were important for an adequate intervention, although adapted, for the case in question, requiring further studies to adapt the systematization directed to care in pediatric nursing.

Key words: Nursing process, Pediatric nursing, Tinea capitis.

1 Universidade do Estado do Pará, Belém - Pará.

*E-mail: brendaramosdesouza@gmail.com.br

${ }^{2}$ Hospital Metropolitano de Urgência e Emergência (HMUE), Ananindeua - PA.

SUBMETIDO EM: 3/2020

ACEITO EM: 4/2020

PUBLICADO EM: 6/2020

REAS/EJCH | Vol.Sup.n.51 | e3505 | DOI: https://doi.org/10.25248/reas.e3505.2020 Página 1 de 9 


\section{RESUMEN}

Objetivo: informar la construcción del proceso de enfermería, a través del Sistematización de la Asistencia de Enfermería desarrollado por el equipo de enfermeras pediátricas para un caso raro de Kerion de Celso, desde su ingreso hasta la transferencia del mismo para la resolución del caso. Informe de experiencia:El primer paso hacia la sistematización de los cuidados de enfermería consistió en la recolección de datos, seguido de un examen físico realizado con un formulario hospitalario. A partir de él, se definieron los problemas activos y potenciales que llevaron a la definición de 6 diagnósticos de enfermería adaptados a la pediatría y las intervenciones dirigidas a actuar sobre los problemas planteados, la evaluación se discutió y revisó diariamente a través de una evolución estandarizada en la Clínica Pediátrica. Consideraciones finales:En este informe, los pasos seguidos para llevar a cabo el proceso de enfermería, a través de la sistematización de la atención de enfermería, fueron importantes para una intervención adecuada, aunque adaptada, para el caso en cuestión, que requieren más estudios para adaptar la sistematización dirigida a la atención en enfermería pediátrica.

Palabras clave: Proceso de enfermería, Enfermería pediátrica, Tiña del cuero cabelludo.

\section{INTRODUÇÃO}

O pensamento científico em enfermagem surgiu com Florence Nightingale em meados do século XIX e suas primeiras iniciativas de conhecimento e registro da frequência das doenças e suas consequências, além de cuidar do ambiente para prevenção de danos. No entanto, somente em 1950 surgiram as discussões acerca da estrutura das atividades de enfermagem em um processo (COSTA FM da, et al., 2014).

Segundo Horta WA e Castellanos BEP (2002), o processo de enfermagem consiste em um modelo didático metodológico voltado à prestação da assistência de enfermagem, com o objetivo de melhor compreender $\mathrm{e}$ organizar aspectos relevantes para a prestação dos cuidados. O Conselho Federal de Enfermagem-COFEN (2009), divide este processo em cinco etapas: coleta de dados, diagnóstico, planejamento e avaliação de enfermagem.

Com o objetivo de operacionalizar o processo de enfermagem, surge a Sistematização da Assistência de Enfermagem (SAE), reunindo recursos humanos, a logística e as ferramentas necessárias para colocar em prática as ações de enfermagem. O termo SAE foi popularizado no Brasil como melhor forma de organização do cuidado em saúde; é uma atividade privativa do enfermeiro, a qual consiste em um conjunto de ações que auxiliam na verificação do problema, intervenção e direcionamento das ações para a prestação de uma assistência eficaz (COFEN, 2009; SANTOS IMF, et al., 2016).

A enfermagem se enquadra na categoria profissional que desenvolve a SAE, com o foco no cuidado. E, independentemente da patologia, este cuidado deve ter como foco as necessidades dos indivíduos (HORTA WA, 1979). Por sua vez, a SAE em pediatria é voltada ao atendimento das necessidades da criança hospitalizada. Portanto, deve ser aplicada pelo enfermeiro responsável pela liderança da equipe de enfermagem, e voltada à efetivação do processo de enfermagem e alcance de metas ao cuidado desenvolvido, em uma cooperação entre a equipe e os pais, familiares e criança (COSTA FM da, et al., 2014).

Nesse sentido, os profissionais de enfermagem também deve estar atento para diversas hipóteses diagnósticas que podem atingir o público infantil no sentido de guiar sua prática de cuidado. Dentre elas, estão as afecções dermatológicas, com especial atenção às dermatofitoses ou infecções do couro cabeludo, que também devem ser tomadas como foco à assistência de enfermagem (HERMANDEZ T, et al., 2004).

Uma das dermatofitoses que mais tem atingido crianças em idade escolar é a causada pela Tinea Capitis (TC). A TC é uma infecção fúngica que se enquadra no conjunto das dermatofitoses que atingem o couro cabeludo, sobrancelhas e cílios, uma vez que agridem principalmente a haste capilar e folículos pilosos, sendo que a sua apresentação clínica varia desde lesões não inflamatórias até inflamatórias agudizadas (SOUISSI A, et al., 2018). 
$\mathrm{Na}$ apresentação clínica não inflamatória da TC, a maior parte das lesões se apresentam de forma avermelhada e descamativa, provocando a perda de cabelos (alopecia) transitória e reversível, semelhante a uma dermatite seborreica em placa arredondada. Em outros casos, geralmente mais raros, essas lesões podem se agravar e agudizar com um padrão inflamatório chamado Kérion Celsi (KC) (HERMANDEZ T, et al.,2004).

As lesões do tipo KC são ocasionadas por uma reação de hipersensibilidade aumentada se comparada a TC. A evolução clínica de tais lesões é exacerbada, uma vez que além da alopecia, apresenta pústulas inflamatórias dolorosas que frequentemente supuram em sua superfície (drenam secreção purulenta), o que facilita a sua transmissibilidade para outras áreas do corpo, além do comprometimento sistêmico, caracterizado por linfonodos infartados e febre que não é verificada na TC comum (PEIXOTO AB, et al., 2012).

A transmissão da TC ocorre através do contato de pessoa para pessoa, contato com animais, solos contaminados e, indiretamente, por objetos contaminados que facilitam sua transmissibilidade (SILVA SF, et al., 2017).

Devido seu alto grau de disseminação, estudos epidemiológicos em Portugal entre 2004 a 2013, identificaram 224 casos de TC que atingiram crianças em idade escolar (menores de 10 anos) e do sexo masculino. No Brasil, a TC não é de notificação compulsória, principalmente por ser tratada como uma doença comum em países tropicais, apesar de apresentar manifestações raras do tipo KC (SABINO R, et al., 2014; BRASIL, 2002).

Embora existam poucos estudos sobre a prevalência da TC no Brasil, em uma revisão de estudos epidemiológicos e de casos no país, foi possível verificar a ocorrência de 1.332 casos distribuídos em diferentes regiões, sendo o maior registro encontrado em Pernambuco.

Por ser uma doença incomum, seu diagnóstico envolve um exame clínico detalhado, além de exames de cultura e micológico direto das lesões. Após diagnóstico, o tratamento pode ser realizado utilizando drogas antifúngicas, sendo a griseofulvina a droga de escolha aliada ao corticoide oral (SILVA CS da, et al., 2019; SILVA SF, et al., 2017).

O diagnóstico e o tratamento precoce auxiliam a resolutividade das lesões supurativas, além de prevenir a disseminação da infecção para outras áreas do corpo e a perda permanente dos cabelos nas áreas afetadas, de forma geral há um bom prognóstico da doença com a cura clínica (ISA-ISA R, ARENAS R, ISA M, 2010).

Dessa forma, o objetivo do artigo foi relatar a construção do processo de enfermagem por meio da SAE desenvolvida pela equipe de enfermeiros da pediatria para um caso raro de $\mathrm{KC}$, desde sua admissão até a transferência do mesmo para resolução do caso.

\section{RELATO DE EXPERIÊNCIA}

A equipe de enfermeiros da pediatria, junto aos residentes e tutores da Residência de Urgência e Emergência da Universidade do Estado do Pará, decidiu aplicar os conhecimentos referentes ao processo de enfermagem para aperfeiçoar a assistência durante o atendimento de um caso raro de KC, associado ao trauma desenvolvido por uma criança, durante suas vivências práticas na Clínica Pediátrica em um hospital de referência em Urgência e Emergência do Norte do Pará.

A criança era um menor de 1 ano e 3 meses, que teve como mecanismo do trauma uma queda da própria altura durante brincadeira com um cachorro; após tratamento, foi liberada para retorno ambulatorial e retornou 11 dias depois, com quadro clínico diferente do inicial. No primeiro atendimento, apresentou edema de face, lesão em região cefálica e febre. Após a re-internação, evoluiu para edema generalizado de face, crânio e região cervical, com aumento do perímetro cefálico em $21 \mathrm{~cm}$, pele seca e descamativa com má higiene corporal.

Progrediu com evolução clínica de anasarca (edema generalizado) e febre. Estes dados foram coletados com a genitora durante a primeira internação na Clínica pediátrica confirmada nos dados do prontuário. A 
mesma relatou histórico de alergia à dipirona, desconhecimento de outras morbidades e outras doenças crônicas desenvolvidas pela família.

A coleta adequada dos dados foi precedida de um exame físico detalhado (Quadro 1), utilizando um formulário padronizado pela clínica com o objetivo de verificar os principais sistemas fisiológicos atingidos, 0 que foi essencial para correlação do quadro clínico e a condução dos diagnósticos.

A tomografia computadorizada foi utilizada como diagnóstico diferencial (onde não foi constado comprometimento neurológico) e o exame micológico direto associado à avaliação clínica dermatológica confirmou a existência de uma infecção fúngica agravada pelo trauma.

Quadro 1 - Achados do exame físico realizado na criança no momento de admissão na Clínica Pediátrica.

\begin{tabular}{|c|l|}
\hline Sistemas & \multicolumn{1}{c|}{ Achados do exame físico } \\
\hline $\begin{array}{c}\text { Percepção e } \\
\text { Cognição }\end{array}$ & - Consciente, choroso, irritado em períodos, face álgica constante; \\
\hline Cabeça & $\begin{array}{l}\text { - Edema em face, áreas de flutuação e coleção em couro cabeludo, lesão ampla e } \\
\text { extensa com necrose seca, com área de descolamento. } \\
\text { - Discreto exsudato purulento em região frontal, em local de exposição de calota } \\
\text { craniana 3 centímetros por 3 centímetros; }\end{array}$ \\
\hline Pescoço & - Nódulos palpáveis; \\
\hline Tórax & $\begin{array}{l}\text { - Simétrico, com expansibilidade preservada, Taquipneia (frequência respiratória } \\
\text { maior que 40 movimentos respiratórios) * transitória em choros; }\end{array}$ \\
\hline Ausculta Pulmonar & - Murmúrios vesiculares; \\
\hline Ausculta Cardíaca & - Normocárdico (em torno de 100 batimentos por minuto), sem ruídos adventícios; \\
\hline Abdômen & - Distendido, ruídos hidroaéreos presentes, sem massas palpáveis e indolor; \\
\hline Genitália & - Dermatite (assadura) em bolsa escrotal e região interglútea; \\
\hline Pele & - Anasarca; Pele seca; \\
\hline Extremidades & - Cateter Venoso em femoral esquerda. \\
\hline
\end{tabular}

Fonte: Souza BR de, et al., 2020, segundo o Caderno de Atenção Básica n³3 do Ministério da Saúde (2012).

Para continuidade do processo de enfermagem, os pesquisadores classificaram os problemas em potenciais e ativos. Os problemas potenciais selecionados foram a idade e uso de polifármacos. A partir do problema da idade, surgiu o risco de queda e de úlcera por pressão (criança em berço).

Como problemas ativos, além da lesão ocasionada pelo KC, foi definida a dor, o edema, a diarreia e a febre como pontos críticos para o caso em questão. A partir destes problemas ativos, surgiram os diagnósticos de integridade da pele prejudicada, dor aguda, risco de desequilíbrio eletrolítico e hipertermia. 
Quadro 2 - Diagnósticos e Prescrições de enfermagem adaptados para o caso.

\begin{tabular}{|c|c|}
\hline $\begin{array}{l}\text { Diagnósticos de } \\
\text { enfermagem }\end{array}$ & Prescrições de Enfermagem e Intervenções de Enfermagem \\
\hline Risco de queda & $\begin{array}{l}\text {-Manter Grades elevadas (checagem } 3 \text { vezes ao dia, com manutenção constante } \\
\text { de toda a equipe de enfermagem); } \\
\text {-Orientar familiar quanto ao risco de queda ( } 2 \text { vezes ao dia); } \\
\text {-Observar estado de consciência (checagem } 3 \text { vezes ao dia); }\end{array}$ \\
\hline $\begin{array}{l}\text { Risco de úlcera } \\
\text { por pressão }\end{array}$ & $\begin{array}{l}\text {-Manter pele hidratada; } \\
\text {-Manter lençol sem dobras para evitar o cisalhamento da pele; } \\
\text {-Mudança de decúbito a cada } 2 \mathrm{~h} ; \\
\text {-Observar e manter cuidados em áreas de pressão; }\end{array}$ \\
\hline Dor aguda & $\begin{array}{l}\text { - Anotar localização, tipo, frequência, intensidade e gravidade da dor (3 vezes ao } \\
\text { dia, de acordo com a escala de face álgica); }\end{array}$ \\
\hline $\begin{array}{l}\text { Integridade da } \\
\text { pele prejudicada }\end{array}$ & $\begin{array}{l}\text {-Observar aspecto da pele (a cada } 8 \text { horas); } \\
\text {-Observar presença de edema (a cada } 8 \text { horas e, principalmente, após o banho); }\end{array}$ \\
\hline $\begin{array}{ll}\text { Risco } & \text { de } \\
\text { desequilíbrio } & \\
\text { eletrolítico } & \end{array}$ & -Anotar débito urinário (a cada troca de fralda ou 3 vezes ao dia); \\
\hline Hipertemia & -Aferir sinais vitais, anotar e comunicar caso haja alteração (a cada 4 horas); \\
\hline
\end{tabular}

Fonte: Souza BR de, et al., 2020, baseado no North American Nursing Diagnosis Association (GARCEZ, et al., 2018) disponível no sistema hospitalar.

O planejamento de enfermagem foi realizado com base nos diagnósticos e prescrições, cujas ações prescritas eram checadas e implementadas pela equipe técnica com a supervisão do enfermeiro. Além dos problemas ativos e potenciais, priorizou-se a lesão fúngica do couro cabeludo, que foi tratada com o banho no leito, curativos oclusivos diários e com técnica asséptica adequada, em concomitância com o tratamento medicamentoso de acordo com a prescrição médica para o caso de KC.

A avaliação do processo de enfermagem era realizada diariamente, geralmente após a visita diária de enfermagem e durante a visita multiprofissional que ocorre uma vez por semana.

A evolução na Clínica Pediátrica foi realizada pelos enfermeiros na periodicidade de uma vez ao dia e sempre quando eram notadas alterações no estado clínico do menor, geralmente após as visitas de enfermagem nos diferentes turnos do dia (Quadro 3).

Dentre as orientações de enfermagem dadas ao familiar, fora priorizada a higiene adequada das mãos, objetos e banho da criança, o que foi essencial para a contenção da disseminação de infecção fúngica para outras áreas do corpo.

Após 7 dias de internação hospitalar foi possível transferir a criança para outra instituição de saúde específica em saúde da mulher e da criança, uma vez que apesar do diagnóstico clínico ter sido feito na instituição do relato, a mesma é voltada apenas para casos de urgência e emergência em trauma e ortopedia não conduzindo demais casos clínicos que não tenham trauma envolvido. 
Quadro 3 - Evolução do plano terapêutico aplicado de acordo com os problemas levantados para o caso clínico do menor, Ananindeua-PA, 2020.

\begin{tabular}{|c|c|c|c|c|}
\hline Datas & $03 / 04$ & $05 / 04$ & $08 / 04$ & $09 / 04$ \\
\hline \multirow{6}{*}{$\begin{array}{l}\text { Problema } \\
\text { ativo }\end{array}$} & \multicolumn{4}{|c|}{ - Lesão com área de necrose; } \\
\hline & \multirow[t]{2}{*}{-Hipertermia } & - & Lesão em face; & Lesão em face \\
\hline & & ------------------------- & Edema Corporal & Edema corporal \\
\hline & -Anasarca; & 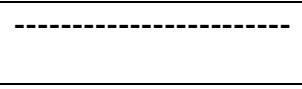 & $\begin{array}{l}\text { Dermatite } \\
\text { amoniacal }\end{array}$ & $\begin{array}{l}\text { Dermatite } \\
\text { Amoniacal }\end{array}$ \\
\hline & \multicolumn{4}{|c|}{-Dor } \\
\hline & \multicolumn{4}{|c|}{-Diarreia } \\
\hline $\begin{array}{l}\text { Problema } \\
\text { potencial }\end{array}$ & \multicolumn{4}{|c|}{$\begin{array}{c}\text {-Uso de dispositivo invasivo; } \\
\text {-Uso de Polifármacos; } \\
\text {-Não conhece suas limitações; } \\
\text {-Idade; } \\
\text {-Acamado; }\end{array}$} \\
\hline \multirow{7}{*}{ Metas } & $\begin{array}{l}\text {-Regressão parcial } \\
\text { de anasarca -04/04; }\end{array}$ & ALCANÇADA & $\begin{array}{l}\text { Controle parcial e } \\
\text { edema - 09/04; }\end{array}$ & $\begin{array}{l}\text { PARCIALMENTE } \\
\text { ALCANÇADA }\end{array}$ \\
\hline & $\begin{array}{l}\text {-Prevenção de } \\
\text { infecção em Cateter } \\
\text { Veoso Central - } \\
\text { 06/04; }\end{array}$ & ALCANÇADA & $\begin{array}{l}\text { Controle diarreia, } \\
\text { avaliar melhora após } \\
\text { término de antibiótico } \\
-10 / 04 ;\end{array}$ & $\begin{array}{l}\text { PARCIALMENTE } \\
\text { ALCANÇADA }\end{array}$ \\
\hline & \multicolumn{3}{|c|}{$\begin{array}{l}\text {-Prevenção de Lesão por pressão e quedas durante o período de } \\
\text { internação; }\end{array}$} & ALCANÇADA \\
\hline & \multicolumn{3}{|c|}{-Controle de dor e agitação excessiva em 48 horas; } & $\begin{array}{l}\text { PARCIALMENTE } \\
\text { ALCANÇADA }\end{array}$ \\
\hline & \multicolumn{3}{|c|}{-Controle da lesão em couro cabeludo -10/04; } & $\begin{array}{l}\text { PARCIALMENTE } \\
\text { ALCANCADA }\end{array}$ \\
\hline & $\begin{array}{l}\text {-Termorregulação } \\
\text {-05/04; }\end{array}$ & ALCANÇADA & $\begin{array}{l}\text { Melhora de dermatite } \\
\text { com epitelização - } 10 / 04\end{array}$ & \begin{tabular}{|l|l|} 
ALCANÇADA \\
\end{tabular} \\
\hline & $\begin{array}{l}\text { Transferência } \\
\text { externa } 10 / 08\end{array}$ & \multicolumn{2}{|l|}{ ALCANÇADA } & \\
\hline \multirow{9}{*}{$\begin{array}{l}\text { Plano de } \\
\text { cuidados }\end{array}$} & \multicolumn{4}{|c|}{-Aplicar pacotes de medidas de quedas e lesão por pressão; } \\
\hline & \multicolumn{4}{|c|}{-Realizar banho no leito diário; } \\
\hline & \multicolumn{4}{|c|}{-Mudança de decúbito a cada 2 horas; } \\
\hline & \multicolumn{4}{|c|}{-Atentar para sinais vitais a cada 4 horas; } \\
\hline & \multicolumn{4}{|c|}{$\begin{array}{l}\text {-Orientar acompanhante sobre a importância da higiene das mãos e do uso de } \\
\text { equipamento de proteção individual } 2 \text { vezes ao dia; }\end{array}$} \\
\hline & \multicolumn{4}{|c|}{-Observar edema e registrar o grau de acordo com teste clínico; } \\
\hline & \multicolumn{4}{|c|}{ - Realizar limpeza ocular 3 vezes ao dia; } \\
\hline & ------------------ & \multicolumn{3}{|c|}{ Trocar a fralda de imediato após a diurese ou evacuação; } \\
\hline & ----------------- & \multicolumn{3}{|c|}{ Aplicar medicação de uso tópico de acordo com prescrição médica; } \\
\hline
\end{tabular}

Fonte: Souza BR de, et al., 2020.

\section{DISCUSSÃO}

Apesar das dermatofitoses do couro cabeludo serem comuns, principalmente as causadas por TC, esta doença tem importante impacto na saúde pública mediante ao seu alto grau de disseminação em crianças na faixa etária escolar, mas também pelo impacto social relevante que a doença gera, uma vez que há a necessidade do afastamento da escola e do convívio com outras crianças. Além de ser facilmente transmissível, esta doença também pode ser confundida com outras infecções fúngicas, o que gera a necessidade de atenção redobrada por parte dos profissionais da saúde que atendem ao público infantil (SABINO R, et al., 2014). O enfermeiro é o profissional responsável por realizar os cuidados diretos à criança hospitalizada e deve estar atenta a prestação de uma assistência que previna, promova e recupere à saúde. 
O mesmo também deve se comprometer com o tratamento da criança, o que foge de um cuidado pautado apenas em saber técnico e prático da assistência, já que é necessário um olhar voltado ao saber científico e cuidado integral, sobretudo quando se trata de doenças raras, que fogem da manifestação clínica padrão e necessitam de maior atenção por parte destes profissionais (FERREIRA TMC, et al., 2016).

Segundo a resolução 358 , de 2009 , do COFEN, o processo de enfermagem se inicia através da coleta de dados, também conhecida com histórico de enfermagem e consiste no levantamento de informações do paciente, família e coletividade, que podem influenciar no quadro clínico do paciente. Neste relato foi verificado que o histórico dos hábitos de higiene e inspeção da pele na calota craniana mostrou como sinal de alerta para um caso atípico de infecção de pele que podem ter sido agravados pelo trauma o que foi essencial para o diagnóstico adequado do caso.

$\mathrm{Na}$ literatura científica, foram achados 2 relatos de casos clínicos que associaram o traumatismo cranioencefálico ao aparecimento da infecção por KC, nestes casos foi observado que a abrasão ou lesão em região cefálica próxima ao couro cabeludo associado aos mau hábito de higiene local, a presença de animais e o solo contaminado, favoreceram para a complicação da TC em ambas as crianças, semelhante 0 que ocorreu neste relato (PEIXOTO AB, et al., 2012; SILVA SF, et al., 2017).

Com a definição clínica, os diagnósticos de enfermagem foram feitos através do North American Nursing Diagnosis Association (NANDA-i), já integrado no sistema on-line hospitalar. Primeiro, foram realizados os diagnósticos relacionados à pele, seguidos dos diagnósticos de enfermagem de: risco de queda e risco de úlcera por pressão relacionada à idade da criança e de dependência dos cuidados da genitora e da equipe, e por fim, definiu-se os diagnósticos prioritários ao estado clínico da patologia: dor aguda, a hipertermia e o risco de desequilíbrio hidroeletrolítico, relacionado ao uso de diuréticos e edema.

Os diagnósticos que mais se relacionam à pele se resumem em três: risco de integridade da pele prejudicada, integridade da pele e tissular prejudicada. Neste estudo, a integridade da pele prejudicada foi destaque devido à apresentação de lesões típicas de $\mathrm{KC}$ em couro cabeludo, por outro lado, apesar de não serem tão comuns se comparadas às doenças respiratórias em crianças, a hipertermia $(15,2 \%)$ e a dor $(7,1 \%)$ ainda se apresentam como sintomas frequentes em afecções dermatológicas infantis (RIBEIRO MAS, LAGES JSS, LOPES MHBM, 2012; ASSIS MN de, et al., 2015).

O diagnóstico de integridade da pele prejudicada está relacionado a um conjunto de fatores que envolvem procedimentos cirúrgicos, mas também doenças dermatológicas e alguns cuidados que devem ser fornecidos para preservação da pele, como mobilidade no leito de forma adequada, higiene corporal, nutrição e realização de procedimentos invasivos em porções não danificadas (LEON PAP de, NÓBREGA MML da, 2012). As intervenções de enfermagem foram baseadas no Nursing Intervention Classification (NIC), adaptados para pediatria, e compreendem as intervenções consideradas no domínio de fisiológicas complexas, na classe de controle de pele e feridas, e controle de perfusão tissular, uma vez que consideraram especificamente os problemas ativos e potenciais (MARQUES DKA, et al., 2014).

Como foi possível verificar, houve limitação dos diagnósticos e intervenções de acordo com o disponível pelo sistema eletrônico hospitalar. Esta dificuldade também foi relata por SOARES e colaboradores (2015). Em outro estudo desenvolvido em uma Clínica pediátrica de São Paulo, foram levantadas 63 intervenções de enfermagem, deste total, somente 4 se enquadram neste estudo: o controle de eliminação (relacionado ao quadro diarreico), controle da imobilidade (criança acamada restrita ao leito), facilitação do autocuidado (relacionado ao cuidado com olhos e banho) e controle de pele e feridas (ASSIS MN de, et al., 2015).

Constatou-se a importância de se documentar e registrar adequadamente as informações. Planejar, mensurar e avaliar as intervenções através de uma estruturação mínima das ações que devem ser executadas para elevação dos padrões de assistência de enfermagem. Demonstrando, também, que a presença de instrumentos padronizados na SAE e no Conjunto Internacional de Dados Mínimos de Enfermagem (i-NMDS) são essenciais para prestação destes cuidados (MARQUES DKA, et al., 2014). Segundo Nóbrega RV, et al. (2011), o processo de identificação de problemas e a construção de diagnósticos de enfermagem que gerem intervenções adequadas para cada caso, é desafiador e varia de acordo com o 
julgamento minucioso por parte do enfermeiro sobre os processos vitais afetados no ser humano. Esse processo torna-se ainda mais difícil quando o público alvo são crianças hospitalizadas que merecem uma atenção especial, com diagnósticos e intervenções de enfermagem adaptados para suas necessidades humanas básicas afetadas.

Neste relato, a coleta de dados, o exame físico estruturado e a evolução se enquadram na definição de dados mínimos a serem coletados para condução adequada de diagnósticos e prescrições essenciais para a realização adequada da SAE, bem como as intervenções foram essenciais para prevenção da ocorrência de problemas potenciais, os quais poderiam ter agravado o estado clínico da criança. Vale ressaltar a necessidade de novos estudos para constituição de instrumentos adequados à SAE em pediatria, assim como um sistema eletrônico mais adequado às prioridades de cada clínica, auxiliando os profissionais no desenvolvimento de uma assistência mais direcionada às necessidades de cada caso.

\section{AGRADECIMENTOS E FINANCIAMENTO}

Agradecemos a toda equipe multiprofissional da Clínica Pediátrica, em especial, à equipe de enfermagem que auxiliou na construção de uma assistência adequada à criança, de forma qualificada e humanizada.

\section{REFERÊNCIAS}

1. ASSIS MN de, et al. Intervenções de enfermagem em pediatria: contribuição para a mensuração da carga de trabalho. Rev. esc. enferm. USP; 2015.

2. BRASIL. Cadernos de Atenção Básica n³3- Saúde da Criança: crescimento e desenvolvimento. Brasília, DF: O ministério, 2012.

3. BRASIL. Departamento de Atenção Básica. Dermatologia na Atenção Básica / Ministério da Saúde, Secretaria de Políticas de Saúde.- 1ª edição. - Brasília: Ministério da Saúde, 2002.

4. COFEN-CONSELHO FEDERAL DE ENFERMAGEM. Resolução COFEN № 358/2009: Sistematização da Assistência de Enfermagem e a implementação do Processo de Enfermagem em ambientes, públicos ou privados, em que ocorre o cuidado profissional de Enfermagem. Brasília: COFEN; 2009.

5. COSTA FM da, et al. Sistematização da Assistência de enfermagem em unidade de internação pediátrica: percepção da equipe de enfermagem. Revista Norte Mineira de enfermagem, 2014; 3 (2): 104-119.

6. FERREIRA TMC, et al. Cuidando da criança hospitalizada com doença de wilson: relato de experiência. Cienc Cuid Saude. 2016; 15(3): 575-581.

7. GARCEZ RM, et al. Diagnósticos de enfermagem da NANDA-l: definições e classificação 2018-2020 .11 ed. Porto Alegre: Artmed, Editado como livro impresso em 2018.

8. HERNÁNDEZ T, et al. Tinhas do Couro Cabeludo na Idade Pediátrica. Nascer e Crescer 2004;13: 23-6.

9. HORTA WA, CASTELLANOS BEP. Processo de enfermagem. São Paulo: EPU; 1979.

10. ISA-ISA R, ARENAS R, ISA M. Inflammatory tinea capitis: kerion, dermatophytic granuloma, and mycetoma, Clin Dermatol, 2010, 28: 133-136.

11. LEON PAP de, NÓBREGA MML da. Diagnóstico de Enfermagem em crianças hospitalizadas utilizando a Nanda -l: estudo de caso. Online Brazilian Journal of Nursing. 2012. 11(1).

12. MARQUES DKA, et al. Conjunto Internacional de Dados Mínimos de Enfermagem: estudo comparativo com instrumentos de uma clínica pediátrica. Rev. bras. enferm. [Internet]. 2014 Aug; 67( 4 ): 588-593.

13. NÓBREGA RV, NÓBREGA MML da, SILVA KL. Diagnósticos, resultados e intervenções de enfermagem para crianças na Clínica Pediátrica de um hospital escola. Rev. bras. enferm. [Internet]. 2011; 64(3): 501-510.

14. PEIXOTO AB, et al. Kerion: a importância da sua diferenciação com infecção bacteriana do couro cabeludo. Relato de caso. Rev Bras Clin Med. São Paulo, 2012; 10(3): 243-5.

15. RIBEIRO MAS, LAGES JSS, LOPES MHBM. Diagnósticos de enfermagem relacionados à pele: definições operacionais. Rev. Latino-Am. Enfermagem. 2012; 20(5).

16. SABINO R, et al. Tinea capitis: análise retrospetiva de casos diagnosticados entre 2004 e 2013 . Boletim Epidemiológico Observações 2014; 3: 26-29.

17. SANTOS IMF, et al. SAE-Sistematização da assistência de enfermagem: guia prático. Salvador: Coren -BA, 2016.

18. SILVA CS da, et al. Etiologia e epidemiologia da tinea capitis: relato de série de casos e revisão da literatura. Revista Brasileira de Análises Clínicas. 2019; 51(1): 9-16. 
19. SILVA SF, et al. Kérion celsi: uma complicação rara da Tinea capitis. Nascer e Crescer [Internet]. 2017; 26(2): 126128.

20. SOARES MI, et al. Sistematização da assistência de enfermagem: facilidades e desafios do enfermeiro na gerência da assistência. Esc Anna Nery, 2015; 19(1): 47-53.

21. SOUISSI A, et al. Morse code-like hairs in tinea capitis disappear after successful treatment. Int. J. Dermatol. 2018. 